\title{
P146 The Predictive Role of Arterial Stiffness in the Development of Acute Kidney Injury in Patients Undergoing Surgical Aortic Valve Replacement
}

Evangelia Sigala*, Charalambos Vlachopoulos, Konstantinos Triantafyllou, Andreas Katsaros, Nikolaos Koumallos, Vasilios Lozos, Nikolaos Baikoussis, Ilias Kouerinis, Nikolaos Giakis, Demosthenous Michael, Dimitrios Terentes Printzios, Konstantinos Filis, Dimitrios Tousoulis

Hippokration Hospital of Athens

\section{ABSTRACT}

Purpose/Background/Objectives: Acute kidney injury (AKI) is a serious postoperative complication. Increased arterial stiffness has been shown to be an independent risk factor for cardiovascular events. Our aim was to investigate whether arterial stiffness is a predictor of AKI in patients following surgical aortic valve replacement (SAVR).

Methods: Eighty-four patients (mean age $72 \pm 8$ years, 34 females) with moderate to severe aortic stenosis undergoing SAVR were included. As indicators of arterial stiffness aortic hemodynamics, carotid-femoral pulse wave velocity (cfPWV) and brachial-ankle pulse wave velocity (baPWV) were assessed prior to surgery. Renal dysfunction was defined when eGFR was below $60 \mathrm{ml} / \mathrm{min}$ ( $n=28,33 \%)$. AKI was defined using KDIQO criteria.

Results: Twelve patients (14\%) developed AKI. There was no significant difference in aortic hemodynamics and cfPWV between the two groups. baPWV significantly correlated with AKI $(r=0.313, p=0.004)$. In logistic regression analysis, increase of baPWV per 1 Standard Deviation (Odds Ratio $[\mathrm{OR}]=2.76,95 \%$ Confidence intervals $[\mathrm{CI}]: 1.25-6.11, p=0.012$ ) and presence of renal dysfunction ( $\mathrm{OR}=14.93,95 \% \mathrm{CI}$ : $2.55-87.32, p=0.003)$ were associated with higher risk for AKI even after adjustment for age, gender, systolic blood pressure and diabetes. baPWV was a stronger predictor of AKI than baseline creatinine (Area under the curve [AUC] $0.68,95 \%$ CI: $0.52-0.84, p=0.05$ vs AUC $0.61,95 \%$ CI: $0.46-0.77, p=0.21 ; p<0.05$ ).

Conclusion: baPWV could be considered as a useful predictive biomarker for AKI after SAVR, especially in patients with renal dysfunction prior to surgery.

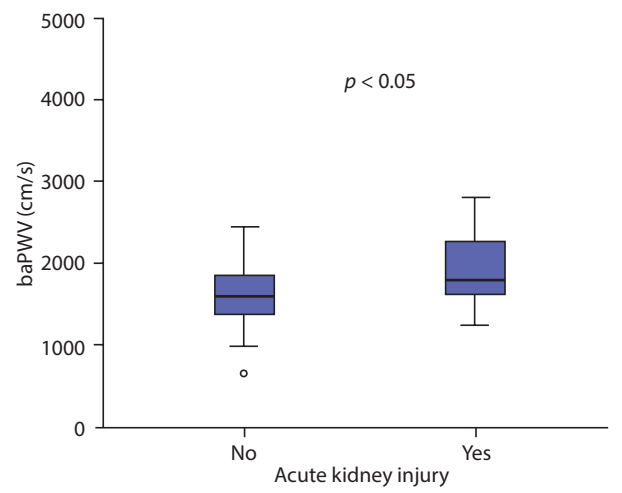

(C) 2019 Association for Research into Arterial Structure and Physiology. Publishing services by Atlantis Press International B.V. This is an open access article distributed under the CC BY-NC 4.0 license (http://creativecommons.org/licenses/by-nc/4.0/). 